\section{Metabolic Basis of Treatment Failures; Autophagy and Malignant Cancer Progression}

\section{Maxwell Omabe $^{1 *}$, Martin Ezeani ${ }^{2}$ and Ugoeze Donatus ${ }^{1}$}

\begin{abstract}
Cells use a number of pathways to generate energy and synthesize cellular building blocks including lipids, carbohydrates and nucleotides. Alterations in metabolic pathways have been implicated in many pathophysiological conditions. This mechanism is exploited by both resident epithelial cancer cells and the fibroblast. Indeed, cancer and normal cells differ dramatically in terms of their energy requirements and use of metabolic pathways. Mitochondrial dysfunction and metabolic impairment contribute immensely to chemotherapeutic resistance and metastatic cancer progression through signaling pathways that revolve around autophagy. The current study reviewed relevant studies that underpin autophagy and highlight the metabolic alterations in the tumour microenvironment that may mediate chemoresistance and malignant cancer progression in both experimental and clinical models. Clearly, autophagy is one of the mechanisms responsible for the refractory response during cancer treatments. Sufficient published studies suggest that autophagy plays a protective role in cancer cells, preventing them from entering the apoptotic pathway, thus contributing to treatment resistance. A better understanding of autophagic role in the process may help in the discovery of new strategies to overcome tumor drug resistance. Here we show that autophagy may represent an important molecular target in development and designing of drug therapy for improving treatment outcome in local and advanced cancer cases.
\end{abstract}

\section{Keywords}

Glycolysis; Oxidative phosphorylation; Cancer progression; Treatment resistance; Autophagy; Metabolism

\section{Introduction}

Two classes of cell death are generally recognized, and include apoptosis and necrosis [1]. Necrosis is defined as accidental form of cell death that occurs in response to acute hypoxic or ischemic injury, such as myocardial infarction and stroke [2]. Other conditions that may result in cells approaching cell death through necrosis include exposure of cells to supraphysiologic conditions like, mechanical force, heat, cold, and membrane-permeabilizing toxins. In necrosis, early loss of integrity of the plasma membrane allows an influx of extracellular ions and fluid, with resultant swelling of the cell and its organelles [2,3]. Autophagy is currently regarded as a third mode of cell death. Thus, cells that do not receive nutrients for extended

*Corresponding author: Maxwell Omabe, Molecular Pathology/Cancer Biology Research Group, Division of Chemical Pathology, Department of Medical Laboratory Science, Faculty of Health Science and Technology, Ebonyi State University Abakaliki, Nigeria, Email: Maswello2002@yahoo.com

Received: March 19, 2013 Accepted: May 23, 2013 Published: May 09, 2013 periods ultimately digest all available substrates and die; this form of cell death is regarded as autophagy-associated cell death.

In Cancer Biology, programmed cell death is a unique feature of cells that is genetically controlled. Apoptosis and autophagy associated cell death are two distinct common forms of cell death $[4,5]$.

In apoptosis, plasma-membrane integrity persists until late in the process. An important feature of apoptosis is cleavage of cytoskeletal proteins by aspartate-specific proteases, which thereby collapse subcellular components and chromatin condensation, nuclear fragmentation, and the formation of plasma-membrane blebs [6,7]. It is generally accepted that more than $50 \%$ of cancer cells have abnormalities involving the apoptotic pathways. Among the best characterized of these abnormalities, are the increased expression of prosurvival BCL2 family proteins and mutations in the tumor-suppressor gene TP53, which encodes tumor protein $\mathrm{p} 53$ (Vazquez et al. 2008). This gene initiates apoptosis in response to DNA damage induced by radiation, chemical agents, oxidative stress, and other agents by transcriptional induction of many proapoptotic proteins, including PUMA, NOXA, and BAX [8]. Proapoptotic and antiapoptotic pathways are strictly controlled by balance between the BCL2 protein family members; this has particular implication on the mitochondrial activities. BCL2, was originally identified as the gene that is commonly altered in the $t(14 ; 18)$ chromosomal translocation in follicular B-cell lymphomas, which inhibits apoptosis and confers cell survival advantage especially to cancer cells [2]. To date, a number of studies have investigated the role of BCL2 expression in different kinds of cancer; results of such studies are specifically applied to further understand why cancer cells often resist chemotherapy. Here, we review current studies that underpin autophagy and highlight the metabolic alterations in the tumour microenvironment that may mediate chemoresistance and malignant cancer progression in both experimental and clinical models.

\section{Autophagy}

Autophagy is a self-digestive process that ensures lysosomal degradation of long-lived proteins and organelles to maintain cellular homeostasis. Clear evidence from published papers suggests that autophagy may be the major cellular pathway for degradation of long-lived proteins and cytoplasmic organelles. It involves the rearrangement of subcellular membranes to sequester cargo for delivery to the lysosome where the sequestered material is degraded and recycled [9]. For many decades, it has been known that autophagy occurs in a wide range of eukaryotic organisms and in multiple different cell types during starvation, cellular and tissue remodeling, and cell death. It is equally a conserved and dynamic process in which portions of the cytoplasm and organelles are sequestered in a doublemembrane vesicle called an autophagosome, which then fuses with the lysosomes, where the captured material is degraded [4].

Figure 1 illustrating role of autophagy and the biochemistry of treatment resistance in tumor cells, especially the fibroblast and epithelial cancer cells [10]. The figure shows a schematic representation of metabolic differences in constituent cells within
ScîTechnol Technology and Medicine 


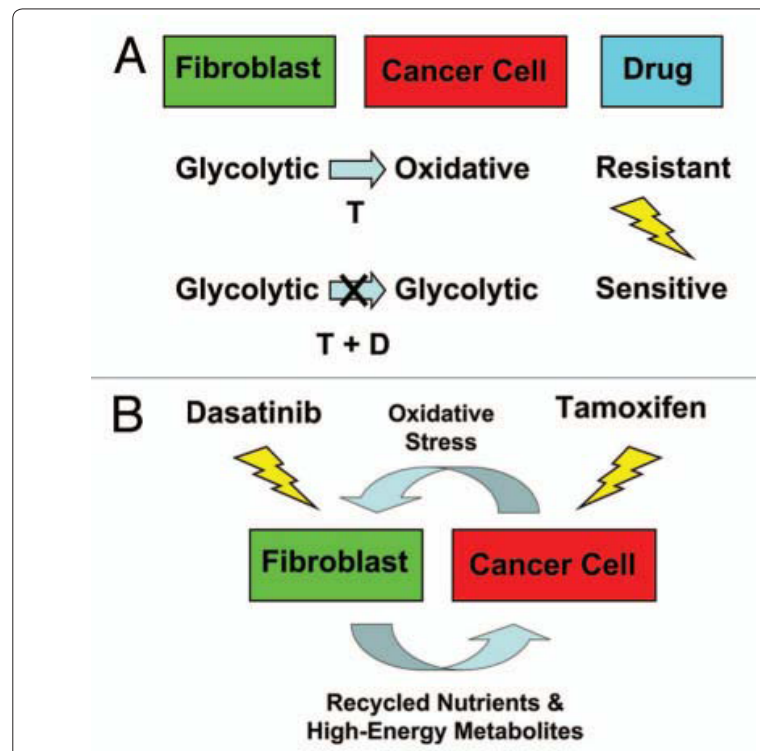

Figure 1: Illustrating role of autophagy and the biochemistry of treatment resistance in tumor cells, especially the fibroblast and epithelial cancer cells.

tumor microenvironment that underpin treatment resistance and loss of apoptosis in cancer cells. Panel A shows that cancer cells exhibit oxidative metabolism and posses increased mitochondrial activities which is strongly supported by the aerobic glycolysis and autophagy in fibroblast. However, Panel B suggests that by reversing the metabolic indices in the tumor cells and the stroma, cancer cells become sensitive to hormone deprivation and chemotherapy; thus a reversed Warburg effect. This means that by inhibiting oxidative or mitochondrial metabolism and inducing glycolysis in the cancer cell (using Dasatinib) hormone deprived breast cancer would respond to treatment

Autophagy serves to degrade structures and recycle their biochemical component for use in energy production and other biosynthetic reactions [11]. The cargo can consist of organelles, protein aggregate, nucleic acids, lipids and as well as pathogens. Autophagosomes fuse with lysosomes to form autolysomes, where the cargo is being degraded. The end products for the digestive process are basic molecular building blocks such as amino acids, fatty acid and nucleotides, which are released back into the cytoplasm by lysosomal permeases [12]. Sufficient data from yeast genetic screens have considerably improved our knowledge about the molecular mechanisms of autophagy, hence, a number of genes involved in fundamental steps of autophagic pathway have been identified [13]. Most of these autophagy genes are present in higher eukaryotes indicating that this process has been evolutionarily conserved. In yeast, autophagy is mainly involved in adaptation to starvation, but in multicellular organisms this route has emerged as a multifunctional pathway involved in a variety of additional processes such as programmed cell death, removal of damaged organelles and development of different tissue-specific functions [8]. Furthermore, autophagy is associated with a growing number of pathological conditions, including cancer, myopathies and neurodegenerative disorders [13].

In humans, genetic studies show that autophagy-related genes are required for lifespan extension. Research evidence currently implicates autophagy in ageing control [14]. Furthermore, results in Drosophila demonstrate that by promoting basal expression of the autophagy gene Atg8 in the nervous system, the lifespan of the organism might be extended by $50 \%$, suggesting that autophagy pathway may contribute in regulating cell death and survival [14]. However, evidence shows that the molecular changes that define cell death by autophagy are completely different from cell death through apoptosis. Experience of the author shows that cancer cells may use autophagy as an adaptive mechanism which allows them to remodel their genome to withstand and survive in a critically stressed tumor microenvironment [14]. In addition, it appears that enhanced aerobic glycolysis and/or autophagy in the tumor stroma may support epithelial cancer cell growth and aggressive behavior, via the secretion of high-energy metabolites. These nutrients include lactate and ketones, as well as chemical building blocks, such as amino acids (glutamine) and nucleotides. Importantly, it is known that lactate and ketones serve as fuel for cancer cell oxidative metabolism, and building blocks that sustain the anabolic needs of rapidly proliferating cancer cells.

Several genetic studies have established the link between the autophagy machinery and tumorigenesis [11]. In fact previous published evidence showed that monoallelic deletion of the essential autophagy regulatory gene Beclin 1 was associated with ovarian, breast, and prostate cancers [15]. Defects in essential autophagy genes Atg5 and Beclin 1 promote tumorigenesis of certain cell lines. Allelic loss of Beclin 1 and deficiencies in expression of two other autophagy-regulating genes, Atg $4 \mathrm{C}$ and Bif-1, render mice prone to tumor development [15].

Moreover, as mentioned above, the regulation of autophagy is tightly controlled by signaling pathways that regulate tumorigenesis. Indeed, inactivating mutations or allelic deletions in tumor suppressor genes whose products positively regulate autophagy (e.g., PTEN, TSC1/TSC2, and p53) are frequently observed in human tumors, emphasizing the tumor-suppressive function of autophagy. On the other hand, some tumors have aberrant activation of genes like $B c l-2$, Akt, Class I PI3K, and NF- $\kappa B$ whose products have tumor promoter functions and repress autophagy [16]. Here we review the role of autophagy in cancer progression.

\section{Warburg effect and autophagy}

In the early 1920s, Otto Warburg, a Nobel Laureate, formulated a hypothesis to explain the "fundamental basis" of cancer, based on his observations that tumors displayed a metabolic shift toward glycolysis [17]. In 1963, Christian de Duve, another Nobel Laureate, first coined the phrase auto-phagy. First, cancer cells are known to operate in a particular state of hypoxia; this often results in production of increased level of hydrogen peroxide [17]. Then, as a consequence, oxidative stress in cancer-associated fibroblasts, drives autophagy, mitophagy, and aerobic glycolysis. In fact, recently published evidence clearly showed the metabolic relationship between cancer cells and the fibroblast in driving tumour progression [18]. Clearly, the authors proposed a new paradigm to explain the compartment-specific role of autophagy in tumor metabolism. In that model, autophagy and mitochondrial dysfunction in the tumor stroma was shown to induce autophagy and promote cellular catabolism; this results in production of recycled nutrients. These chemical building blocks and high-energy "fuels" would then drive the anabolic growth of tumors, via autophagy resistance and oxidative mitochondrial metabolism in cancer cells. 
Clearly this new form of stromal-epithelial metabolic coupling: "twocompartment tumor metabolism model [18] may explain why cancer cells resist chemotherapy and apoptosis. In a mechanistic study, Salem and colleague [18] genetically created autophagic fibroblasts which were generated by allowing the cells to stably overexpress key target genes that lead to AMP-kinase activation, such as such as damage-regulated autophagy modulator (DRAM) and liver kinase B1 (LKB1; a critical regulator of cellular stress and the autophagic response) [18]. In addition, autophagy-resistant cancer cells were also derived by over expressing GOLPH3, which functionally promotes mitochondrial biogenesis. The experiments provided data which strongly support Warburg effect, suggesting that autophagic fibroblasts expressed mitochondrial dysfunction, with increased production of mitochondrial fuels (L-lactate and ketone body accumulation). However, in the same study, GOLPH3 overexpressing breast cancer cells were autophagy-resistant, and exhibited signs of increased mitochondrial biogenesis and function, which resulted in increased tumor growth. Thus, autophagy in the tumor stroma and oxidative mitochondrial metabolism (OXPHOS) in cancer cells may both dramatically promote tumor growth, independent of tumor angiogenesis [18]. It is known that GOLPH3 is a mitochondrial protein which critically regulates mitochondrial lipid biogenesis by shuttling between the Golgi apparatus culminating in an increase in the delivery of mitochondrial phospho-lipids (such as cardiolipin) which would result in overall increase in mitochondrial mass [18]. The precise impact of autophagy on malignant transformation has not yet been clarified, but it appears that this complex process might be mainly directed by metabolisms in the different cell types in the tumor microenvironment. Thus, relationship between autophagy and cancer progression under the influence of the tumor microenvironment may indicate a novel aspect in cancer chemotherapy.

\section{Metabolic basis of drug resistance: the role of Autophagy}

There are sufficient published evidence establishing that metabolic paradigm in the tumour microenvironment, specifically the stroma and the cancer cells may synergistically promote cancer malignant progression and tumour growth; this has been suggested as the leading cause of treatment failures in endocrine cancers including prostate and breast cancer. Evidence has shown that glycolytic fibroblasts protect cancer cells against apoptosis, by providing a steady nutrient stream to mitochondria in cancer cells as described above. Currently, there are merging experimental data suggesting that the explained metabolic differences commonly referred to as the Warburg and the reversed Warburg effect may underlay chemoresistance and metastatic progression in advanced and localized solid tumour. Martinez-Outschoorn and colleague (20) investigated the molecular basis of tamoxifen-resistance in $\mathrm{ER}(+)$ breast cancer cells and showed that MCF7 cells were Tamoxifen-sensitive, but became resistant when co-cultured with hTERT-immortalized human fibroblasts. The authors introduced a drug combination (Tamoxifen + Dasatinib) that could over-come fibroblast-induced Tamoxifen-resistance in breast cancer cells. Clearly, this drug combination acutely induced the Warburg effect (aerobic glycolysis) in MCF7 cancer cells; this abruptly cut off the ability of the epithelia cancer cells to use their fuel supply, resulting in effective killing of the cancer cells. That study demonstrated that Warburg effect in tumor cells is not the cause of cancer, but rather, it may provide the necessary clues to preventing chemo-resistance in cancer cells. Furthermore, the study clearly showed that autophagy represents a potential molecular target in cancer cells. In fact, by combing Tamoxifen and Dasatinib a bioreductive effect was observed on both co-cultured fibroblasts and cancer cells alike, potentially reducing tumor-stroma co-evolution. This is in line with substantial published and unpublished data which demonstrate that by targeting hypoxia chemoresistant cancer cells could be reverted. From available evidence, it is now clear that chemo-resistance in both metabolic and stromal can be overcome by targeting mitochondrial function in epithelial cancer cells [19]. Thus, simultaneously targeting both (1) autophagy in the tumor stroma and (2) mitochondrial function in epithelial cancer cells, with combination therapies, a novel treatment regimen that may provide a successful approach to anti-cancer therapy may be designed.

\section{Hypoxia mediates autophagy induced cancer progression}

Hypoxia induces cancer progression by regulating various aspect of cancer biology such as angiogenesis, glucose metabolism, iron metabolism and cancer cell proliferation [20,21]. Hypoxia is a know activator of autophagy and this is mediated through hypoxia inducible factor induction of BNIP3 and BNIP3L via their BH3 domain [20]. However, the clear signaling pathway of this process is not understood. Emerging evidence now suggests that hypoxia may induce autophagic degradation of Cav-1 in stromal fibroblasts; this process is completely blocked by the autophagy inhibitor chloroquine. For instance, hypoxia-induced degradation of Cav-1, resulted in upregulation of a number of well-established autophagy/ mitophagy markers, including LC3, ATG16L, HIF-1 $\alpha$ and NFkB in the study of Martinez-Outschoorn et al. 2010. Thus, it appears that oxidative stress mediated induction of HIF1- and NFkB-activation in fibroblasts drives the autophagic degradation of Cav-1.

Martinez-Outschoorn and colleague [22] strongly showed that an acute knock-down of Cav-1 in stromal fibroblasts, using an siRNA approach, was sufficient to induce autophagy, with the upregulation of both lysosomal and mitophagy markers. The question remains, how do loss of stromal Cav-1 and the induction of stromal autophagy affect cancer cell survival or loss of apoptosis? Interestingly, the authors demonstrated that a loss of Cav-1 in stromal fibroblasts protects adjacent cancer cells against apoptotic cell death [22]. Thus, autophagic cancer-associated fibroblasts, in addition to providing recycled nutrients for cancer cell metabolism, also play a protective role in preventing the death of adjacent epithelial cancer cells [19]. In another experiment, fat pads derived from Cav-1 (-/-) null mice showed a hypoxia-like response in vivo with upregulation of autophagy markers, such as LC3 and BNIP3L [22]. Clearly, loss of stromal fibroblast Cav-1 is a biomarker for chronic hypoxia, oxidative stress and autophagy in the tumor microenvironment, consistent with its ability to predict early tumor recurrence, lymph node metastasis and tamoxifen-resistance in human breast cancers [22]. This implies that cancer patients with defects in stromal Cav-1 may benefit from HIF-inhibitors or bioreductive therapies [23] as well as autophagy/ lysosomal inhibitors. In addition, another study also demonstrated that loss of stromal Cav-1 expression and HIF1-alpha-activation was associated with driving cancer-associated fibroblast phenotype, through the paracrine production of nutrients via autophagy and aerobic glycolysis [24].

However, it remains unknown if HIFla-activation is sufficient to confer the cancer-associated fibroblast metabolic phenotype that supports cancer progression [21,23]. A recent mechanistic study demonstrated that fibroblasts harboring activated HIF1a 
showed a dramatic reduction in Cav-1 levels and a shift towards aerobic glycolysis, as evidenced by a loss of mitochondrial activity, and an increase in lactate production, including BNIP3 and BNIP3L expression, markers indicating autophagic destruction of mitochondria [24]. Most importantly, fibroblasts expressing activated HIF1a increased tumor mass by $\sim 2$-fold and tumor volume by $\sim 3$-fold, lymph node metastasis, without a significant increase in tumor angiogenesis [24]. Hypoxia is a generally accepted inducer of HIF activation. Therefore, activated HIFla may be sufficient to functionally confer cancer-associated fibroblast phenotype. It is also known that HIFla expression is required for induction of autophagy in cancer cells. Based on available published data, it appears that autophagy in cancer-associated fibroblasts promotes tumor growth via the paracrine production of recycled nutrients, which directly support cancer cells development. Conversely, autophagy in cancer cells represses tumor growth. Taken together, Hypoxia may mediate autophagic tumor Stroma model of cancer metabolism which results in increased cell survival and chemoresistance.

Emerging studies have further provided experimental evidence that provides more molecular clues to support the suggestion that metabolic alterations in stroma glycolysis may promote tumorigenesis. Infact, in a xenograft model, when normal human fibroblasts were genetically-engineered to express the two isoforms of pyruvate kinase M (PKM1 and PKM2), a key enzyme in the glycolytic pathway, fibroblasts expressing PKM1 or PKM2 greatly promoted the growth of co-injected MDA-MB-231 breast cancer cells, without an increase in tumor angiogenesis [25]. Interestingly, PKM1 and PKM2 promoted tumorigenesis by different mechanism(s). Expression of PKM1 enhanced the glycolytic power of stromal cells, with increased output of lactate [25]. Analysis of tumor xenografts demonstrated that PKM1 fibroblasts greatly induced tumor inflammation, as judged by CD45 staining [26]. In contrast, PKM2 did not lead to lactate accumulation, but triggered a "pseudo-starvation" response in stromal cells, with induction of an NFkB-dependent autophagic program, and increased output of the ketone body 3-hydroxyburyrate. Strikingly, in situ evaluation of Complex IV activity in the tumor xenografts demonstrated that stromal PKM2 expression drives mitochondrial respiration specifically in tumor cells [25]. Finally, immuno-histochemistry analysis of human breast cancer samples lacking stromal Cav-1 revealed PKM1 and PKM2 expression in the tumor stroma [25]. Put together, these studies suggest that a subset of human breast cancer patients with a loss of stromal Cav-1 show profound metabolic changes in the tumor microenvironment. As such, this subgroup of patients may benefit therapeutically from potent inhibitors targeting glycolysis, autophagy and/or mitochondrial activity (such as metformin).

\section{Autophagy as a Drug Target for Cancer Treatment}

The goal of anticancer therapy is to effectively compromise tumor cell growth and survival, so as to cause cancer regression and prevent (or at least delay) cancer recurrence, thus improving patient quality of life and survival $[10,26]$. Apoptosis is commonly inactivated in cancer, often in association with disease progression, and renders tumor resistant to chemotherapy and radiation induced cell death, undisputedly contributing to treatment resistance and earlier patient demise [27]. Despite recent advances in cancer treatment, many tumors still exhibit unsatisfactory responsiveness to biological agent, chemotherapy and/or radiation, either reoccurring or continuing to grow during or after treatment [28]. Up-regulation of autophagy is a common occurrence in response to cancer therapy, expected to occur in both tumor and normal cells, but likely playing a more critical role in the survival of the already metabolically stressed cancer cells, as explained above, and thus contributing to treatment resistance. At the same time, however, this prosurvival function of autophagy provides a novel therapeutic opportunity, as concurrent autophagy inhibition may preferentially sensitize tumor cells to anticancer agent by depriving them of an essential survival mechanism that may be dispensable for normal cell viability under similar conditions [29].

\section{Autophagy inducers in cancer therapy}

Excessive or sustained autophagy has the potential to induce tumor cell death and therefore, be a potential strategy for cancer treatment [30]. Although autophagy inhibitors combined with standard treatment are emerging as promising anticancer agents, certain cancer cells and xenograft tumors were sensitized to therapeutic regimens involving autophagy induction rather than inhibition. This was commonly observed in an apoptosis-defective background, where cancer cells were committed to non-apoptotic cell death modes, including necrosis, necroptosis and possibly autophagic cell death [31].

Autophagy is regulated by the mTOR pathway and mTOR inhibitors can activate autophagy. Rapamycin is a naturally occurring mTOR inhibitor and its analog temsirolimus (Cc1-779), everolimus (RAD-001) and deforolimus (AP-23 573) selectively target mTorc1 to stimulate autophagy. With the exception of renal cell and neuroendocrine carcinomas and lymphomas, rapamycin and its analogs have had limited success [32, 33]. A potential explanation for their modest anti tumor activity is the inability of rapamycin to inhibit mTORc2 and its ability to abrogate the S6k1- mediated negative feedback loop to the p13k-AKT pathway that result in rebound AKT activation [29]. The goal of achieving a more complete blockade of the mTOR pathway has led to the development of ATP competitive mTOR inhibitors of both mTORC1 and mtorc2 (e.g. PP242, AZD80 55, WYE1 32) and the dual P13k - mTOR inhibitor NVP-BEZ235 that also inhibit P13k. the limited activity of mTOR inhibitor as monotherapy has led to the evaluation of drug combination. Rapamycin combined with cytotoxic chemotherapy was shown to enhance apoptosis in vitro and to enhance antitumor efficacy in vivo [30].

Another inducer of autophagy is called imatinib, which is a Bcr-abl tyrosine kinas inhibitor (TKi). It is effective against chronic myelogenous leukemia (CML). Imatinib has been shown to modulate autophagy through the regulation of lysosomal component [34]. Other autophagy modulators include antidepressant such as the selective serotonin reuptake inhibitor, fliuoxetine and the norepinephrine reuptake inhibitor, meprotiline. Both drugs were shown to induce autophagy in chemoresistent Burkitt lymphoma [35]. The anti-diabetic, biguanide drug metformin has been shown to inhibit mTOR signaling through it upstream mediator, AMPK [36]. Metformin has cytostatic effect on a variety of cancer cells types. In prostate cancer cell, metformin inhibited 2 deoxy glucose induced autophagy, decreased Beclin 1 expression and triggered a switch from cell survival to cell death [36].

\section{Autophagy inhibitors in cancer therapy}

Autophagy inhibitors can be broadly classified as early versus late stage inhibitors of the pathway. Early stage inhibitors include 
3- methyadenine (3 MA), wortmaninin and Ly294002 that target the class $111 \mathrm{p} 13 \mathrm{k}$ (VPS34), while late stage inhibitors include chloroquine (CQ), or hydrochlorquine (HCQ), bafilomycin A1 and monensin that prevent fusion of autophagosomes with the lysosomes [37,38]. Bafilomycin A1 is a specific inhibitor of vacuolar AT pase while monensin and CQ/HCQ are lysomotropic drugs that prevent the acidification of lysosomal compartment. The inhibitor of autophagy combined with chemotherapy may enhance treatment efficacy by inhibiting stress adaptation and increasing cell death. Of the known autophagy inhibitors, only CQ/HCQ has been evaluated in human given their common usage as anti malarial drugs and in autoimune disorder. HCQ is prefered to CQ in human given its more favorable side effect profile [39]. The combination of CQ and HDAC inhibitor vorinostat was also shown to significantly reduce tumor burden and to induce apoptosis in colon cancer zenograft model, Similarly, CQ in combination with saracatinib, a SRC inhibitor, produced a 2 -food increase in apoptotic tumor cells compared to saracatinib alone [40]. Protein degradation occurs through autophagy in lysosomes, but also within proteasome for ubiquitinated proteins.

\section{Conclusion}

Based on available published data, it appears that autophagy in cancer-associated fibroblasts promotes tumor growth via the paracrine production of recycled nutrients, which directly support cancer cells development. Conversely, autophagy in cancer cells represses tumor growth. Taken together, Hypoxia may mediate autophagic tumor Stroma model of cancer metabolism which results in increased cell survival and chemoresistance and loss of stromal fibroblast Cav1 is a biomarker for oxidative stress and autophagy in the tumor microenvironment, this is consistent with its ability to predict early tumor recurrence, lymph node metastasis and tamoxifen-resistance in human breast cancers [22]. Put together, these studies suggest that a subset of human cancer patients with a loss of stromal Cav-1 may show profound metabolic changes in the tumor microenvironment. It appears that enhanced aerobic glycolysis and/or autophagy in the tumor stroma may support epithelial cancer cell growth and aggressive behavior, via the secretion of high-energy metabolites. These nutrients include lactate and ketones, as well as chemical building blocks, such as amino acids (glutamine) and nucleotides. Therefore, subgroup of cancer patients may benefit therapeutically from potent inhibitors targeting glycolysis, autophagy and/or mitochondrial activity (such as metformin). On the whole, autophagy plays a role in promoting cancer progression and a number of combined therapies that alter the Warburg effect in the tumour stroma and epithelia cancer cells may abrogate the tumor progression.

\section{References}

1. Laster SM, Wood JG, Gooding LR (1988) Tumor necrosis factor can induce both apoptotic and necrotic forms of cell lyses. J Immunol 141: 2629-2634.

2. Luke CJ, Pak SC, Askew YS, Naviglia TL, Askew DJ, et al. (2007) An intracellular serpin regulates necrosis by inhibiting the induction and sequelae of lysosomal injury. Cell 130: 1108-1119.

3. Youle RJ, Strasser A (2008) The BCL-2 protein family: opposing activities that mediate cell death. Nat Rev Mol Cell Biol 9: 47-59.

4. Klionsky DJ, Emr SD (2000) Autophagy as a regulated pathway of cellular degradation. Science 290: 1717-1721.

5. Kroemer G, Galluzzi L, Vandenabeele P, Abrams J, Alnemri ES, et al. (2009) Classification of cell death: recommendations of the Nomenclature Committee on Cell Death. Cell Death Differ 16: 3-11.
6. Malhi H, Gores GJ, Lemasters JJ (2006) Apoptosis and necrosis in the liver: a tale of two deaths? Hepatology 43: S31-S44.

7. Ben Sahra I, Laurent K, Giuliano S, Larbret F, Ponzio G, et al. (2010) Targeting cancer cell metabolism the combination of metformin and 2-deoxy glucose induces P53-dependent apoptosis in prostate cancer cells. Cancer Res 70: 2465-2475.

8. Vazquez A, Bond EE, Levine AJ, Bond GL (2008) The genetics of the p53 pathway, apoptosis and cancer therapy. Nat Rev Drug Discov 7: 979-987.

9. Levine B, Klionsky D. (2004) Development by self-digestion: molecular mechanisms and biological functions of autophagy. Dev Cell Apr 6: 463-477.

10. Chen N, Karantza-Wadswarth V (2009) Role and Regulation of Autophagy in cancer. Biochim Biophys Acta 1793: 1516-1523.

11. Levine B, Kroemer G (2008) Autophagy in the pathogenesis of disease. Cell 132: $27-42$.

12. Liou W, Geuze HJ, Geelen MJ, Slot JW (1997) The autophagic and endocytic pathways converge at the nascent autophagic vacuoles. J Cell Biol 136: 6170

13. Mariño G, López-Otín C (2004) Autophagy: molecular mechanisms physiological functions and relevance in human pathology. Cell Mol Life Sci 61: 1439-1454.

14. Korolchuk VI, Mansilla A, Menzies FM, Rubinsztein DC (2009) Autophagy inhibition compromises degradation of ubiquitin-proteasome pathway substrates. Mol Cell 33: 517-527.

15. Liang XH, Jackson S, Seaman M, Brown K, Kempkes B, et al. (1999) Induction of autophagy and inhibition of tumorigenesis by beclin 1. Nature 402: 672-676.

16. White E, DiPaola RS (2009) The double-edged sword of autophagy modulation in cancer. Clin Cancer Res 15: 5308-5316.

17. Vaupel P, Kallinowski F, Okunieff P (1989) Blood flow, oxygen and nutrient supply, and metabolic microenvironment of human tumors: a review. Cancer Res 49: 6449-6465.

18. Salem AF, Whitaker-Menezes D, Lin Z, Martinez-Outschoorn UE, Tanowitz $\mathrm{HB}$, et al. (2012) Two-compartment tumor metabolism: autophagy in the tumor microenvironment and oxidative mitochondrial metabolism (OXPHOS) in cancer cells. Cell Cycle 11: 2545-2556.

19. Martinez-Outschoorn UE, Lin Z, Ko YH, Goldberg AF, Flomenberg N, et al. (2011) Understanding the metabolic basis of drug resistance: therapeutic induction of the Warburg effect kills cancer cells. Cell Cycle 10: 2521-2528.

20. Majmundar AJ, Wong WJ, Simon MC (2010). Hypoxia inducible factors and the response to hypoxic stress. Mol Cell 40: 294-309.

21. Omabe M, Ezeani M (2011) Infection, Inflammation and Prostate Carcinogenesis. Journal of Infection, Genetics and Evolution 11: 1195-1198.

22. Martinez-Outschoorn UE, Trimmer C, Lin Z, Whitaker-Menezes D, Chiavarina B, et al. (2010) Autophagy in cancer associated fibroblasts promotes tumor cell survival: Role of hypoxia, HIF1 induction and NFKB activation in the tumor stromal microenvironment. Cell Cycle 9: 3515-3533.

23. Omabe M, Onyeanusi JC, Ezeani M, Amos N, Okekpa SI (2012) Gene expression profile, androgen independence and prostate cancer. Journal of Cancer Therapy 3: 637-644.

24. Chiavarina B, Whitaker-Menezes D, Migneco G, Martinez-Outschoorn UE, Pavlides S, et al. (2010) HIF1-alpha functions as a tumor promoter in cancer associated fibroblasts, and as a tumor suppressor in breast cancer cells: Autophagy drives compartment-specific oncogenesis. Cell Cycle 9: 35343551.

25. Chiavarina B, Whitaker-Menezes D, Martinez-Outschoorn UE, Witkiewicz AK, Birbe R, et al. (2011) Pyruvate kinase expression (PKM1 and PKM2) in cancer-associated fibroblasts drives stromal nutrient production and tumor growth. Cancer Biol Ther 12: 1101-1113.

26. Kondo Y, Kanzawa T, Sawaya R, Kondo S (2005) The role of autophagy in cancer development and response to therapy. Nat Rev Cancer 5: 726-734.

27. Høyer-Hansen $M$, Jäättelä M (2008) Autophagy: an emerging target for cancer therapy. Autophagy 4: 574-580. 
28. Platini F, Pérez-Tomás R, Ambrosio S, Tessitore L (2010) Understanding autophagy in cell death control. Curr Pharm Des 16: 101-113.

29. Yogalingam G, Pendergast AM (2008) Abl kinases regulate autophagy by promoting the trafficking and function of lysosomal components. J Biol Chem 283: 35941-35953.

30. Meric-Bernstam F, Gonzalez-Angulo AM (2009) Targeting the mTOR signaling network for cancer therapy. J Clin Oncol 27: 2278-2287.

31. Boya P, Gorizalez-Angulo AM, Casares N, Perfettini JL, Dessen P, et al (2005) Inhibition of Macroautophagy Triggers Apoptosis. Mol Cell Biol 25 : 1025-1040.

32. Abraham RT, Gibbons JJ (2007) The mammalian target of rapamycin signaling pathway: twists and turns in the road to cancer therapy. Clin Cancer Res 13: 3109-3114.

33. O'Reilly KE, Rojo F, She QB, Solit D, Mills GB, et al. (2006) mTOR inhibition induces upstream receptor tyrosine kinase signaling and activates Akt. Cancer Res 66: 1500-1508

34. Cloonan SM, Williams DC (2010) The antidepressants maprotiline and fluoxetine induce Type II autophagic cell death in drug-resistant Burkitt's lymphoma. Int J Cancer 128: 1712-1723.
35. Dowling RJ, Zakikhani M, Fantus IG, Pollak M, Sonenberg N (2007) Metformin inhibit mammalian target of rampamycin dependent translation initiation in breast cancer cells. Cancer Res 67: 10804-10812.

36. Bellot G, Raguel GI, Pierre G, Johana C, Nathalie M (2009) Hypoxia induced Autophagy is medicated through hypoxia -inducible factor induction of BNip3 and BNiP32 via their BH3 domain. Mol Cell Biol 29: 2570-2581.

37. Carew JS, Nawrocki ST, Kahue CN, Zhang H, Yang C, et al. (2007). Targeting autophagy augments the anticancer activity of the histone deacetylase inhibitor SAHA to overcome Bcr-Abl-mediated drug resistance. Blood 110: 313-322.

38. Ruiz-Irastorza G, Ramos-Casals $M$, Brito-Zeron $P$, Khamashta MA (2010) Clinical efficacy and side effects of antimalarials in systemic lupus erythematosus: a systematic review. Ann Rheum Dis 69: 20-28.

39. Wu Z, Change PC, Yang JC, Chu CY, Wang LY, et al. (2010) Autophagy Blockade Sensitizes Prostate Cancer Cells towards Src Family Kinase Inhibitors. Genes Cancer 1: 40-49.

40. Simon MC (2006) Coming up for air: Hif -1 and mitochondrial oxygen consumption. Cell Metab 3: 150-151.

\section{Author Affiliations}

${ }^{1}$ School of Biomedical Science, Faculty of Health Science, Ebonyi State University, Nigeria

${ }^{2}$ Department of Neurosciences, University of Sussex, United Kingdom
Submit your next manuscript and get advantages of SciTechnol submissions

* 50 Journals

* 21 Day rapid review process

- 1000 Editorial tean

2 Million readers

* More than 5000 facebogk

* Publication immediately after acceptance

* Quality and quick editorial, review processing

Submit your next manuscript at • www.scitechnol.com/submission 